\title{
On operator-valued cosine sequences on UMD spaces
}

\author{
by
}

Wojciech Chojnacki (Adelaide and Warszawa)

\begin{abstract}
A two-sided sequence $\left(c_{n}\right)_{n \in \mathbb{Z}}$ with values in a complex unital Banach algebra is a cosine sequence if it satisfies $c_{n+m}+c_{n-m}=2 c_{n} c_{m}$ for any $n, m \in \mathbb{Z}$ with $c_{0}$ equal to the unity of the algebra. A cosine sequence $\left(c_{n}\right)_{n \in \mathbb{Z}}$ is bounded if $\sup _{n \in \mathbb{Z}}\left\|c_{n}\right\|$ $<\infty$. A (bounded) group decomposition for a cosine sequence $c=\left(c_{n}\right)_{n \in \mathbb{Z}}$ is a representation of $c$ as $c_{n}=\left(b^{n}+b^{-n}\right) / 2$ for every $n \in \mathbb{Z}$, where $b$ is an invertible element of the algebra (satisfying $\sup _{n \in \mathbb{Z}}\left\|b^{n}\right\|<\infty$, respectively). It is known that every bounded cosine sequence possesses a universally defined group decomposition, the so-called standard group decomposition. Here it is shown that if $X$ is a complex UMD Banach space and, with $\mathscr{L}(X)$ denoting the algebra of all bounded linear operators on $X$, if $c$ is an $\mathscr{L}(X)$-valued bounded cosine sequence, then the standard group decomposition of $c$ is bounded.
\end{abstract}

1. Introduction. Given a strongly continuous one-parameter group $\{G(t)\}_{t \in \mathbb{R}}$ acting on a Banach space $X$, it is immediate that

$$
C(t)=\frac{1}{2}(G(t)+G(-t))
$$

is a strongly continuous cosine function, that is, a strongly continuous operator-valued solution of the cosine functional equations:

(i) $C(s+t)+C(s-t)=2 C(s)(t)$ for any $s, t \in \mathbb{R}$,

(ii) $C(0)=I_{X}$,

where $I_{X}$ denotes the identity operator on $X$. One of the central themes in the theory of cosine functions is whether a representation of the above form, analogous to the equality $\cos a t=\left(e^{i a t}+e^{-i a t}\right) / 2$, exists for a given cosine function [1, Section 3.16], [3], [12], [13, Section 2.5], [14, Sections III.6 and III.8], [17, [19, Section III.1.1], [21]. Recently Haase [15], improving on a result of Cioranescu and Keyantuo [9], showed that every strongly continuous cosine function acting on a complex UMD space has a group decomposition generated by a bounded strongly continuous one-parameter group. In

2010 Mathematics Subject Classification: 39B42, 47A60, 47D03, 47D09.

Key words and phrases: cosine sequence, cosine function, group decomposition, UMD space, transference method. 
this note, a complement to [8] in which the group decomposability of cosine sequences was discussed, we establish an analogue of Haase's result for operator-valued cosine sequences. The starting point for our work is the fact that every bounded cosine sequence with values in a complex unital Banach algebra admits a universally defined group decomposition generated by an invertible element of the algebra. This so-called standard group decomposition may or may not be bounded itself, that is, the invertible element generating the group decomposition may or may not be doubly power bounded. The existence of a standard group decomposition is specific to cosine sequences and does not have a counterpart for cosine functions; in fact, a bounded strongly continuous one-parameter cosine family of operators may fail to have any group decomposition whatsoever [7, 16, 18]. The main result of this paper asserts that if a cosine sequence is formed by operators acting on a complex UMD space and is bounded, then the standard group decomposition for this cosine sequence is bounded. This result confirms the naturality and effectiveness of the concept of standard group decomposition in dealing with problems concerning bounded cosine sequences.

2. Background. In this section we shall give some basic definitions and results that will be needed throughout.

2.1. Cosine sequences. Let $\mathbf{A}$ be a complex Banach algebra with a unity $e$ and a norm $\|\cdot\|$. A two-sided sequence $c=\left(c_{n}\right)_{n \in \mathbb{Z}}$ with values in $\mathbf{A}$ is called a cosine sequence, or a discrete cosine function, if

(i) $c_{n+m}+c_{n-m}=2 c_{n} c_{m}$ for any $n, m \in \mathbb{Z}$,

(ii) $c_{0}=e$.

Every cosine sequence $c$ is even: the equality $c_{-n}=c_{n}$ holds for all $n \in \mathbb{Z}$. Furthermore, every cosine sequence $c$ is uniquely determined by its element indexed by 1 , namely,

$$
c_{n}=T_{|n|}\left(c_{1}\right) \quad(n \in \mathbb{Z}),
$$

where, for $n \in \mathbb{N} \cup\{0\}, T_{n}(x)$ is the $n$th Chebyshev polynomial of the first kind

$$
T_{n}(x)=\sum_{k=0}^{[n / 2]}\left(\begin{array}{c}
n \\
2 k
\end{array}\right) x^{n-2 k}\left(x^{2}-1\right)^{k} .
$$

The element $c_{1}$ is commonly termed the generator of $c$. Every element of $\mathbf{A}$ generates a unique cosine sequence. The cosine sequence generated by $a \in \mathbf{A}$ is given by $c_{n}(a)=T_{|n|}(a)$ for $n \in \mathbb{Z}$ and is denoted $c(a)$.

An A-valued cosine sequence $\left(c_{n}\right)_{n \in \mathbb{Z}}$ is bounded whenever $\sup _{n \in \mathbb{Z}}\left\|c_{n}\right\|$ $<\infty$. For an $\mathbf{A}$-valued bounded cosine sequence $c=\left(c_{n}\right)_{n \in \mathbb{Z}}$, we let $\|c\|_{\infty}$ $=\sup _{n \in \mathbb{Z}}\left\|c_{n}\right\|$. 
Let Inv $\mathbf{A}$ be the group of invertible elements of $\mathbf{A}$. It is readily verified that, for each $b \in \operatorname{Inv} \mathbf{A}$, the sequence $\underline{c}(b)$ defined by

$$
\underline{c}_{n}(b)=\frac{1}{2}\left(b^{n}+b^{-n}\right) \quad(n \in \mathbb{Z})
$$

is a cosine sequence. A group decomposition for an A-valued cosine sequence $c=\left(c_{n}\right)_{n \in \mathbb{Z}}$ is a representation of $c$ in the form

$$
c=\underline{c}(b)
$$

for some $b \in \operatorname{Inv} \mathbf{A}$. In view of the uniqueness property of cosine sequences, for (2.1) to hold it is necessary and sufficient that

$$
c_{1}=\underline{c}_{1}(b)=\frac{1}{2}\left(b+b^{-1}\right) .
$$

The element $b$ in 2.1 is referred to as the generator of the corresponding group decomposition. If $b$ is doubly power bounded, i.e, if $\sup _{n \in \mathbb{Z}}\left\|b^{n}\right\|<\infty$, then the group decomposition is termed bounded and $b$ is said to generate a bounded group decomposition.

Suppose that $a \in \mathbf{A}$ generates a bounded cosine sequence $c(a)$. Then the series $\sum_{n \in \mathbb{Z}}\left(4 n^{2}-1\right)^{-1} c_{2 n}(a)$ is absolutely convergent and the following two elements of $\mathbf{A}$ can be defined:

$$
\phi(a)=-\frac{2}{\pi} \sum_{k \in \mathbb{Z}} \frac{1}{4 n^{2}-1} c_{2 n}(a), \quad \psi(a)=a+i \phi(a) .
$$

It turns out that $\psi(a)$ is invertible, has $a-i \phi(a)$ for its inverse, and generates a group decomposition for $c(a)$ [8, Theorem 3.1]. This group decomposition is the standard group decomposition for $c(a)$. The above general construction is perhaps best illustrated by considering a $\mathbb{C}$-valued bounded cosine sequence of the form $c=(\cos n t)_{n \in \mathbb{Z}}$, where $t \in[0,2 \pi)$. In this case $a=\cos t$ alongside

$$
\begin{aligned}
& \phi(a)=-\frac{2}{\pi} \sum_{n \in \mathbb{Z}} \frac{1}{4 n^{2}-1} \cos 2 n t=\sqrt{1-\cos ^{2} t}=|\sin t|, \\
& \psi(a)=\cos t+i|\sin t|,
\end{aligned}
$$

and it is clear that $\psi(a)$ generates a bounded group decomposition for $c$.

2.2. UMD spaces. Throughout the paper, all Banach spaces will be assumed complex. Let $X$ be a Banach space. Let $(\Omega, \mathscr{F}, \mathrm{P})$ be a probability space, and let $\left(\mathscr{F}_{n}\right)_{n \in \mathbb{N}}$ be a non-decreasing sequence of sub- $\sigma$-algebras of $\mathscr{F}$. A sequence $\left(d_{n}\right)_{n \in \mathbb{N}}$ of Bochner integrable $X$-valued random variables is a martingale difference sequence relative to the filtration $\left(\mathscr{F}_{n}\right)_{n \in \mathbb{N}}$ if for all $n \in \mathbb{N}, d_{n}$ is $\mathscr{F}_{n}$-measurable and $\mathrm{E}\left(d_{n+1} \mid \mathscr{F}_{n}\right)=0$, where $\mathrm{E}(\cdot \mid \mathscr{G})$ denotes 
conditional expectation given the sub- $\sigma$-algebra $\mathscr{G}$ of $\mathscr{F}$; the latter condition can be equivalently restated as

$$
\int_{A} d_{n+1} d \mathrm{P}=0
$$

for all $A \in \mathscr{F}_{n}$. Let $L^{p}(\Omega, X)$ denote the set of all $X$-valued strongly measurable functions $f$ on $(\Omega, \mathscr{F}, \mathrm{P})$ such that $\int_{\Omega}\|f(\omega)\|^{p} d \mathrm{P}(\omega)<\infty$, with the norm

$$
\|f\|_{p, X}=\left(\int_{\Omega}\|f(\omega)\|^{p} d \mathrm{P}(\omega)\right)^{1 / p} .
$$

The Banach space $X$ is UMD (unconditional for martingale differences) if for some (equivalently, all) $1<p<\infty$, there exists a positive constant $C_{p, X}$ such that, for all $n \in \mathbb{N}$,

$$
\left\|\sum_{k=1}^{n} \epsilon_{k} d_{k}\right\|_{p, X} \leq C_{p, X}\left\|\sum_{k=1}^{n} d_{k}\right\|_{p, X}
$$

for every $X$-valued sequence of martingale differences $\left(d_{k}\right)_{k \in \mathbb{N}}$ and for every sequence $\left(\epsilon_{k}\right)_{k \in \mathbb{N}}$ with $\epsilon_{k}= \pm 1$. The class of UMD spaces contains the $L^{p}$ spaces of an arbitrary measure for $1<p<\infty$ and their non-commutative analogues, including the von Neumann-Schatten $p$-classes for $1<p<\infty$, and is stable under the formation of closed subspaces, quotients, and duals. Furthermore each UMD space is reflexive. For the theory of UMD spaces we refer the reader to [4, 5] and references given therein.

In this note the UMD spaces will appear in connection with a specific operator-valued multiplier transform. For $1 \leq p<\infty$, let $l^{p}(\mathbb{Z}, X)$ denote the space of all $X$-valued two-sided absolutely $p$-summable sequences, that is,

$$
l^{p}(\mathbb{Z}, X)=\left\{\eta=\left(\eta_{n}\right)_{n \in \mathbb{Z}} \in X^{\mathbb{Z}} \mid \sum_{n \in \mathbb{Z}}\left\|\eta_{n}\right\|^{p}<\infty\right\}
$$

with the norm

$$
\|\eta\|_{p, X}=\left(\sum_{n \in \mathbb{Z}}\left\|\eta_{n}\right\|^{p}\right)^{1 / p}
$$

When $X=\mathbb{C}$, we abbreviate $l^{p}(\mathbb{Z}, X)$ to $l^{p}(\mathbb{Z})$ and $\|\cdot\|_{p, X}$ to $\|\cdot\|_{p}$. Given $\xi=\left(\xi_{k}\right)_{k \in \mathbb{Z}} \in l^{2}(\mathbb{Z})$, we denote by $\widehat{\xi}$ the Fourier transform of $\xi$ - that is, the element of $L^{2}([0,2 \pi))$ defined by

$$
\widehat{\xi}(t)=\sum_{k \in \mathbb{Z}} \xi_{k} e^{-i k t}
$$

for almost every (a.e.) $t \in[0,2 \pi)$, with the right-hand side understood as 
the limit in the $L^{2}$-norm of the sequence $\left(d_{n}(\xi)\right)_{n \in \mathbb{N}}$ in $L^{2}([0,2 \pi))$ given by

$$
d_{n}(\xi)(t)=\sum_{k=-n}^{n} \xi_{k} e^{-i k t} \quad(t \in[0,2 \pi)) .
$$

Let $f:[0,2 \pi) \rightarrow \mathbb{R}$ be given by

$$
f(t)= \begin{cases}1 & \text { if } 0 \leq t<\pi \\ -1 & \text { if } \pi \leq t<2 \pi\end{cases}
$$

Since $f$ is bounded, Plancherel's theorem ensures the existence of a bounded linear operator $M_{f}: l^{2}(\mathbb{Z}) \rightarrow l^{2}(\mathbb{Z})$ satisfying

$$
\widehat{M_{f} \xi}=f \widehat{\xi} \quad\left(\xi \in l^{2}(\mathbb{Z})\right) .
$$

Since, in addition, $f$ is of bounded variation, it follows from a result of Stechkin [20] (cf. also [11, Theorem 6.4.4]) that $f$ is a $p$-multiplier for each $1<p<\infty$-there exists a positive number $m_{p}$ such that

$$
\left\|M_{f} \xi\right\|_{p} \leq m_{p}\|\xi\|_{p} \quad\left(\xi \in l^{2}(\mathbb{Z}) \cap l^{p}(\mathbb{Z})\right) .
$$

The estimate $(2.3)$ together with $l^{2}(\mathbb{Z}) \cap l^{p}(\mathbb{Z})$ being dense in $l^{p}(\mathbb{Z})$ allows $M_{f}$ to be uniquely extended to a bounded linear operator from $l^{p}(\mathbb{Z})$ into itself, also denoted $M_{f}$, of norm $\leq m_{p}$.

Given a linear space $\ell$ of complex-valued two-sided sequences, we shall treat the algebraic tensor product $\ell \otimes X$ as a linear space of $X$-valued twosided sequences, with a typical element

$$
\sum_{k=1}^{n} \xi_{k} \otimes x_{k} \quad\left(\xi_{1}, \ldots, \xi_{n} \in \ell, x_{1}, \ldots, x_{n} \in X\right)
$$

of $\ell \otimes X$ identified with the $X$-valued two-sided sequence $\eta=\left(\eta_{l}\right)_{l \in \mathbb{Z}}$ given by

$$
\eta_{l}=\sum_{k=1}^{n}\left(\xi_{k}\right)_{l} x_{k} \quad(l \in \mathbb{Z}) .
$$

With this convention, $l^{p}(\mathbb{Z}) \otimes X$ is a subspace of $l^{p}(\mathbb{Z}, X)$ for $1 \leq p<\infty$. Assuming that $1<p<\infty$, consider the tensor product

$$
M_{f, X}=M_{f} \otimes I_{X}
$$

as an operator acting on $l^{p}(\mathbb{Z}) \otimes X$; in other words, if $\eta=\sum_{k=1}^{n} \xi_{k} \otimes x_{k} \in$ $l^{p}(\mathbb{Z}) \otimes X$, then $M_{f, X} \eta \in l^{p}(\mathbb{Z}) \otimes X$ is given by

$$
M_{f, X} \eta=\sum_{k=1}^{n} M_{f} \xi_{k} \otimes x_{k} .
$$

A fact of relevance to this paper will be that if $X$ is a UMD space, then $M_{f, X}$ can be extended to a bounded linear operator on $l^{p}(\mathbb{Z}, X)$ [2, Scholium 3.1]. Since $l^{p}(\mathbb{Z}) \otimes X$ is dense in $l^{p}(\mathbb{Z}, X)$, the extension is necessarily unique and 
hereafter it will also be denoted $M_{f, X}$. The operator $M_{f, X}$ acting on $l^{p}(\mathbb{Z}, X)$ is an instance of an operator-valued $p$-multiplier.

3. Standard contraction cosine sequences. Let $X$ be a Banach space and let $\mathscr{L}(X)$ denote the algebra of all bounded linear operators on $X$, with identity $I_{X}$. Let $1 \leq p<\infty$, and, for each $n \in \mathbb{Z}$, let $\mathrm{T}_{n}$ be the translation operator on $l^{p}(\mathbb{Z}, X)$ defined by

$$
\left(\mathrm{T}_{n} \eta\right)_{k}=\eta_{k+n} \quad\left(\eta=\left(\eta_{l}\right)_{l \in \mathbb{Z}} \in l^{p}(\mathbb{Z}, X), k \in \mathbb{Z}\right) .
$$

The operator $\mathbf{T}_{n}$ is a surjective linear isometry and its inverse is $\mathbf{T}_{-n}$. The companion operator $\mathrm{C}_{n}$ defined by

$$
\mathrm{C}_{n}=\frac{1}{2}\left(\mathrm{~T}_{n}+\mathrm{T}_{-n}\right)
$$

is a contraction. The sequence $\mathrm{C}=\left(\mathrm{C}_{n}\right)_{n \in \mathbb{Z}}$ is an $\mathscr{L}(X)$-valued bounded cosine sequence, with $\|\mathrm{C}\|_{\infty}=1$; it will be termed the standard contraction cosine sequence on $l^{p}(\mathbb{Z}, X)$.

Strictly speaking, the notation $\mathrm{T}_{n}, \mathrm{C}_{n}, \mathrm{C}$ is not precise, since it does not indicate on which of the $l^{p}$ spaces these objects are being considered. However, as this will be specified in each result, no confusion should arise.

We now present a result that will form the basis for the subsequent development.

Theorem 3.1. Let $1<p<\infty$ and let $X$ be a UMD Banach space. Then the standard group decomposition of the standard contraction cosine sequence $\mathrm{C}$ on $l^{p}(\mathbb{Z}, X)$ is bounded.

Proof. We denote the generator of the standard group decomposition of C, $\psi\left(\mathrm{C}_{1}\right)$, by $\mathrm{B}$. To prove that $\mathrm{B}$ is doubly power bounded, we first observe that, for each $\eta \in\left(l^{2}(\mathbb{Z}) \cap l^{p}(\mathbb{Z})\right) \otimes X$,

$$
\widehat{\mathrm{C}_{1} \eta}(t)=(\cos t) \widehat{\eta}(t)
$$

for a.e. $t \in[0,2 \pi)$. Here, for $\eta$ represented as

$$
\eta=\sum_{k=1}^{n} \xi_{k} \otimes x_{k} \quad\left(\xi_{1}, \ldots, \xi_{n} \in l^{2}(\mathbb{Z}) \cap l^{p}(\mathbb{Z}), x_{1}, \ldots, x_{n} \in X\right),
$$

$\widehat{\eta}$ denotes the Fourier transform of $\eta$ - the element of $L^{2}([0,2 \pi)) \otimes X$ defined by

$$
\eta=\sum_{k=1}^{n} \widehat{\xi_{k}} \otimes x_{k}
$$

With the aid of 2.2$)$, it is easily verified that

$$
\widehat{\mathrm{B} \eta}(t)=(\cos t+i|\sin t|) \widehat{\eta}(t)
$$


for a.e. $t \in[0,2 \pi)$. Using the operator-valued $p$-multiplier $M_{f, X}$ from the previous section, we define two (projection) operators $P^{ \pm}$in $\mathscr{L}\left(l^{p}(\mathbb{Z}, X)\right)$ by

$$
P^{ \pm}=\frac{1}{2}\left(I_{l^{p}(\mathbb{Z}, X)} \pm M_{f, X}\right) .
$$

Clearly, we have $P^{+}+P^{-}=I_{l^{p}(\mathbb{Z}, X)}$, and $\widehat{P^{+} \eta}=1_{[0, \pi)} \widehat{\eta}$ and $\widehat{P^{-} \eta}=1_{[\pi, 2 \pi)} \widehat{\eta}$ for $\eta \in\left(l^{2}(\mathbb{Z}) \cap l^{p}(\mathbb{Z})\right) \otimes X$. In view of 3.1 , if $n \in \mathbb{Z}$ and $\eta \in\left(l^{2}(\mathbb{Z}) \cap\right.$ $\left.l^{p}(\mathbb{Z})\right) \otimes X$, then

$$
\begin{aligned}
& \widehat{\mathrm{B}^{n} P^{+}} \eta(t)=(\cos t+i \sin t)^{n} 1_{[0, \pi)}(t) \widehat{\eta}(t)=e^{i n t} \widehat{P^{+}} \eta(t)=\widehat{\mathrm{T}_{n} P^{+}} \eta(t), \\
& \widehat{\mathrm{B}^{n} P^{-}} \eta(t)=(\cos t-i \sin t)^{n} 1_{[\pi, 2 \pi)}(t) \widehat{\eta}(t)=e^{-i n t} \widehat{P^{-}} \eta(t)=\widehat{\mathrm{T}_{-n} P^{+}} \eta(t)
\end{aligned}
$$

for a.e. $t \in[0,2 \pi)$. Hence, taking into account that $\left(l^{2}(\mathbb{Z}) \cap l^{p}(\mathbb{Z})\right) \otimes X$ is dense in $l^{p}(\mathbb{Z}, X)$, we infer that $\mathrm{B}^{n} P^{+}=\mathrm{T}_{n} P^{+}$and $\mathrm{B}^{n} P^{-}=\mathrm{T}_{-n} P^{-}$, and further

$$
\left\|\mathrm{B}^{n}\right\| \leq\left\|\mathrm{B}^{n} P^{+}\right\|+\left\|\mathrm{B}^{n} P^{-}\right\|=\left\|\mathrm{T}_{n} P^{+}\right\|+\left\|\mathrm{T}_{-n} P^{-}\right\|=\left\|P^{+}\right\|+\left\|P^{-}\right\| .
$$

Thus B is doubly power bounded.

4. Transference result. To make use of the result from the previous section, we shall need a variant of the transference method of Calderón [6] and Coifman and Weiss [10. This technique, in varying forms, transfers operators affiliated with groups, along with their bounds, to spaces in which the groups act. Here we establish a relevant transference result in the form of a discrete analogue of a transference result of Haase [15, Theorem 3.1].

Let $l_{\mathrm{e}}^{1}(\mathbb{Z})$ be the Banach space of all even sequences in $l^{1}(\mathbb{Z})$. For each $n \in \mathbb{Z}$, let $\delta_{n}$ be the two-sided sequence with all entries equal to 0 except for the $n$th entry which is equal to 1 , and let

$$
\epsilon_{n}=\frac{1}{2}\left(\delta_{n}+\delta_{-n}\right)
$$

With the convolution

$$
(s * t)_{n}=\sum_{m \in \mathbb{Z}} s_{n-m} t_{m} \quad\left(s, t \in l_{\mathrm{e}}^{1}(\mathbb{Z})\right)
$$

as product and with $\epsilon_{0}\left(=\delta_{0}\right)$ as unity, $l_{\mathrm{e}}^{1}(\mathbb{Z})$ is a unital Banach algebra.

Let $\mathbf{A}$ be a complex Banach algebra with a unity $e$, and let $c=\left(c_{n}\right)_{n \in \mathbb{Z}}$ be an $\mathbf{A}$-valued bounded cosine sequence. For each $s=\left(s_{n}\right)_{n \in \mathbb{Z}} \in l_{\mathrm{e}}^{1}(\mathbb{Z})$, we define $\pi_{c}(s) \in \mathbf{A}$ by

$$
\pi_{c}(s)=\sum_{n \in \mathbb{Z}} s_{n} c_{n} .
$$

It is easily verified that

$$
\pi_{c}: l_{\mathrm{e}}^{1}(\mathbb{Z}) \rightarrow \mathbf{A}, \quad s \mapsto \pi_{c}(s),
$$


is a representation of the convolution algebra $l_{\mathrm{e}}^{1}(\mathbb{Z})$, that is,

(i) $\pi_{c}(s * t)=\pi_{c}(s) \pi_{c}(t)$ for any $s, t \in l_{\mathrm{e}}^{1}(\mathbb{Z})$,

(ii) $\pi_{c}\left(\epsilon_{0}\right)=e$.

Moreover, $\pi_{c}$ is continuous, with $\left\|\pi_{c}\right\| \leq\|c\|_{\infty}$.

TheOREM 4.1. Let $X$ be a Banach space, let $c=\left(c_{n}\right)_{n \in \mathbb{Z}}$ be an $\mathscr{L}(X)$ valued bounded cosine sequence, and let $1 \leq p<\infty$. Then, with $\mathrm{C}$ the standard contraction cosine sequence on $l^{p}(\mathbb{Z}, X)$,

$$
\left\|\pi_{c}(s)\right\| \leq 12\|c\|_{\infty}^{2}\left\|\pi_{C}(s)\right\|
$$

for each $s \in l_{\mathrm{e}}^{1}(\mathbb{Z})$.

Proof. Let $s=\left(s_{n}\right)_{n \in \mathbb{Z}}$ be an element of $l_{\mathrm{e}}^{1}(\mathbb{Z})$ with compact support, say in $\{-N,-N+1, \ldots, N-1, N\}$, where $N \in \mathbb{N}$; in other words, $s_{n}=0$ for each $n$ with $|n|>N$. Then, for each $x \in X$ and each $k \in \mathbb{Z}$,

$$
\begin{aligned}
c_{k} \pi_{c}(s) x & =\sum_{l \in \mathbb{Z}} s_{l} c_{k} c_{l} x=\frac{1}{2} \sum_{l \in \mathbb{Z}} s_{l}\left(c_{k+l}+c_{k-l}\right) x \\
& =\frac{1}{2} \sum_{l=-N}^{N} s_{l}\left(c_{k+l}+c_{k-l}\right) x .
\end{aligned}
$$

For each $m \in \mathbb{N}$, we define an operator $i_{m}: X \rightarrow l^{p}(\mathbb{Z}, X)$ by

$$
\left(i_{m} x\right)_{k}=\left\{\begin{array}{ll}
c_{k} x & \text { if }|k| \leq m, \\
0 & \text { otherwise }
\end{array} \quad(x \in X, k \in \mathbb{Z}) .\right.
$$

Clearly, if $k$ is in the range $|k| \leq n, l$ is in the range $|l| \leq N$, and $x \in X$, then

$$
\frac{1}{2}\left(c_{k+l}+c_{k-l}\right) x=\left(\mathrm{C}_{l} i_{n+N} x\right)_{k} .
$$

Consequently, for each $k$ with $|k| \leq n$,

$$
c_{k} \pi_{c}(s) x=\sum_{l=-N}^{N} s_{l}\left(C_{l} i_{n+N} x\right)_{k}=\sum_{l \in \mathbb{Z}} s_{l}\left(C_{l} i_{n+N} x\right)_{k}=\left(\pi_{\mathrm{C}}(s) i_{n+N} x\right)_{k} .
$$

As $I_{X}=2 c_{k}^{2}-c_{2 k}$ for each $k \in \mathbb{Z}$, we have

$$
x=\frac{2}{2 n+1} \sum_{k=-n}^{n} c_{k}^{2} x-\frac{1}{2 n+1} \sum_{k=-n}^{n} c_{2 k} x
$$

for all $n \in \mathbb{N}$. Substituting $\pi_{c}(s) x$ for $x$ in this last identity and taking into account that, in view of $4.2, c_{k} \pi_{c}(s) x=\left(\pi_{\mathrm{C}}(s) i_{2 n+N} x\right)_{k}$ whenever 
$|k| \leq 2 n$, we see that, for each $n \in \mathbb{N}$,

$$
\begin{aligned}
\pi_{c}(s) x & =\frac{2}{2 n+1} \sum_{k=-n}^{n} c_{k}^{2} \pi_{c}(s) x-\frac{1}{2 n+1} \sum_{k=-n}^{n} c_{2 k} \pi_{c}(s) x \\
& =\frac{2}{2 n+1} \sum_{k=-n}^{n} c_{k}\left(\pi_{C}(s) i_{2 n+N} x\right)_{k}-\frac{1}{2 n+1} \sum_{k=-n}^{n}\left(\pi_{C}(s) i_{2 n+N} x\right)_{2 k}
\end{aligned}
$$

For each $n \in \mathbb{N}$, we define an operator $P_{n}: l^{p}(\mathbb{Z}, X) \rightarrow X$ by

$$
P_{n} \eta=\frac{2}{2 n+1} \sum_{k=-n}^{n} c_{k} \eta_{k}-\frac{1}{2 n+1} \sum_{k=-n}^{n} \eta_{2 k} \quad\left(\eta=\left(\eta_{k}\right) \in l^{p}(\mathbb{Z}, X)\right) .
$$

We can now rewrite 4.3 as

$$
\pi_{c}(s)=P_{n} \pi_{\mathrm{C}}(s) i_{2 n+N} .
$$

With this identity in hand, establishing (4.1) is a matter of suitably estimating the norms of $P_{n}$ and $i_{2 n+N}$.

We have, for each $x \in X$,

$$
\left\|i_{2 n+N} x\right\|_{p, X}=\left(\sum_{k=-(2 n+N)}^{2 n+N}\left\|c_{k} x\right\|^{p}\right)^{1 / p} \leq(4 n+2 N+1)^{1 / p}\|c\|_{\infty}\|x\|
$$

so

$$
\left\|i_{2 n+N}\right\| \leq(4 n+2 N+1)^{1 / p}\|c\|_{\infty} .
$$

Let $q$ be the conjugate index of $p$, defined by $1 / p+1 / q=1$. By Hölder's inequality, for each $\eta=\left(\eta_{k}\right) \in l^{p}(\mathbb{Z}, X)$,

$$
\begin{aligned}
\left\|P_{n} \eta\right\| & \leq \frac{2\|c\|_{\infty}}{2 n+1} \sum_{k=-n}^{n}\left\|\eta_{k}\right\|+\frac{1}{2 n+1} \sum_{k=-n}^{n}\left\|\eta_{2 k}\right\| \\
& \leq \frac{2\|c\|_{\infty}+1}{2 n+1} \sum_{k=-2 n}^{2 n}\left\|\eta_{k}\right\| \\
& \leq \frac{2\|c\|_{\infty}+1}{2 n+1}(4 n+1)^{1 / q}\|\eta\|_{p, X} \\
& \leq \frac{2\|c\|_{\infty}+1}{n+1}(4 n+4)^{1 / q}\|\eta\|_{p, X} \\
& =\left(2\|c\|_{\infty}+1\right) 4^{1 / q}(n+1)^{-1 / p}\|\eta\|_{p, X}
\end{aligned}
$$

so

$$
\left\|P_{n}\right\| \leq\left(2\|c\|_{\infty}+1\right) 4^{1 / q}(n+1)^{-1 / p}
$$


Now, on account of 4.4 4 4.6) and $1 \leq\|c\|_{\infty}$,

$$
\begin{aligned}
\left\|\pi_{c}(s)\right\| & \leq\left(2\|c\|_{\infty}+1\right) 4^{1 / q}(n+1)^{-1 / p}(4 n+2 N+1)^{1 / p}\|c\|_{\infty}\left\|\pi_{\mathrm{C}}(s)\right\| \\
& \leq\left(2\|c\|_{\infty}+1\right) 4^{1 / q}(n+1)^{-1 / p}(4 n+4 N+4)^{1 / p}\|c\|_{\infty}\left\|\pi_{\mathrm{C}}(s)\right\| \\
& \leq\left(2\|c\|_{\infty}+1\right) 4\left(1+\frac{N}{n+1}\right)^{1 / p}\|c\|_{\infty}\left\|\pi_{\mathrm{C}}(s)\right\| \\
& \leq 12\|c\|_{\infty}\left(1+\frac{N}{n+1}\right)^{1 / p}\|c\|_{\infty}\left\|\pi_{\mathrm{C}}(s)\right\| .
\end{aligned}
$$

Letting $n \rightarrow \infty$, we deduce 4.1).

In the last step, we remove the support restriction on $s$. For $s=\left(s_{n}\right) \in$ $l_{\mathrm{e}}^{1}(\mathbb{Z})$ and $N \in \mathbb{N}$, let $s^{(N)}$ be the element of $l_{\mathrm{e}}^{1}(\mathbb{Z})$ given by

$$
\left(s^{(N)}\right)_{k}=\left\{\begin{array}{ll}
s_{k} & \text { if }|k| \leq N, \\
0 & \text { otherwise }
\end{array} \quad(k \in \mathbb{Z}) .\right.
$$

For each $N \in \mathbb{N}, s^{(N)}$ has support in $\{-N,-N+1, \ldots, N-1, N\}$, and so (2.1) holds with $s$ replaced by $s^{(N)}$. Furthermore, $\left(s^{(N)}\right)_{N \in \mathbb{N}}$ converges in norm to $s$, and hence also $\left(\pi_{c}\left(s^{(N)}\right)\right)_{N \in \mathbb{N}}$ and $\left(\pi_{C}\left(s^{(N)}\right)\right)_{N \in \mathbb{N}}$ converge in norm to $\pi_{c}(s)$ and $\pi_{\mathrm{C}}(s)$, respectively. This immediately implies (4.1) in the general case.

5. Main result. We are now in a position to establish the central result of the paper.

THEOREM 5.1. The standard group decomposition of an $\mathscr{L}(X)$-valued bounded cosine sequence, where $X$ is a UMD Banach space, is bounded.

Proof. Let $X$ be a UMD Banach space and let $c=\left(c_{n}\right)_{n \in \mathbb{Z}}$ be an $\mathscr{L}(X)$ valued bounded cosine sequence. Define two elements $b_{+}$and $b_{-}$of $l_{\mathrm{e}}^{1}(\mathbb{Z})$ by

$$
b_{ \pm}=\epsilon_{1} \mp \frac{2 i}{\pi} \sum_{k \in \mathbb{Z}} \frac{1}{4 k^{2}-1} \epsilon_{2 k} .
$$

Observe that $\pi_{c}\left(\epsilon_{1}\right)=c_{1}$, that the generator of the standard group decomposition for $c, \psi\left(c_{1}\right)$, coincides with $\pi_{c}\left(b_{+}\right)$, and that $\pi_{c}\left(b_{-}\right)$is the inverse of $\pi_{c}\left(b_{+}\right)$. Select $1<p<\infty$ arbitrarily and let $C$ be the standard contraction cosine sequence on $l^{p}(\mathbb{Z}, X)$. As above, note that $\pi_{\mathrm{C}}\left(\epsilon_{1}\right)=\mathrm{C}_{1}$, that the generator of the standard group decomposition for $\mathbf{C}, \psi\left(\mathbf{C}_{1}\right)$, coincides with $\pi_{\mathrm{C}}\left(b_{+}\right)$, and that $\pi_{\mathrm{C}}\left(b_{-}\right)$is the inverse of $\pi_{\mathrm{C}}\left(b_{+}\right)$. Now, for each $n \in \mathbb{N}$,

$$
\pi_{c}\left(b_{ \pm}\right)^{n}=\pi_{c}\left(b_{ \pm}^{* n}\right) \quad \text { and } \quad \pi_{\mathrm{C}}\left(b_{ \pm}\right)^{n}=\pi_{\mathrm{C}}\left(b_{ \pm}^{* n}\right),
$$

where

$$
s^{* n}=\underbrace{s * \cdots * s}_{n \text { times }}
$$


for any $s \in l_{\mathrm{e}}^{1}(\mathbb{Z})$. This and Theorem 4.1 imply that

$$
\left\|\pi_{c}\left(b_{ \pm}\right)^{n}\right\|=\left\|\pi_{c}\left(b_{ \pm}^{* n}\right)\right\| \leq 12\|c\|_{\infty}^{2}\left\|\pi_{\mathrm{C}}\left(b_{ \pm}^{* n}\right)\right\|=12\|c\|_{\infty}^{2}\left\|\pi_{\mathrm{C}}\left(b_{ \pm}\right)^{n}\right\|
$$

for each $n \in \mathbb{N}$. But, by Theorem 3.1, $\sup _{n \in \mathbb{N}}\left\|\pi_{C}\left(b_{ \pm}\right)^{n}\right\|<\infty$. Therefore we also have $\sup _{n \in \mathbb{N}}\left\|\pi_{c}\left(b_{ \pm}\right)^{n}\right\|<\infty$, and so $\pi_{c}\left(b_{+}\right)$is doubly power bounded.

\section{References}

[1] W. Arendt, C. J. K. Batty, M. Hieber, and F. Neubrander, Vector-Valued Laplace Transforms and Cauchy Problems, Monogr. Math. 96, Birkhäuser, Basel, 2001.

[2] E. Berkson and T. A. Gillespie, Spectral decompositions and harmonic analysis on UMD spaces, Studia Math. 112 (1994), 13-49.

[3] A. Bobrowski, The Widder-Arendt theorem on inverting of the Laplace transform, and its relationships with the theory of semigroups, Methods Funct. Anal. Topology 3 (1997), 1-39.

[4] D. L. Burkholder, A geometrical characterization of Banach spaces in which martingale difference sequences are unconditional, Ann. Probab. 9 (1981), 997-1011.

[5] - Martingales and singular integrals in Banach spaces, in: Handbook of the Geometry of Banach Spaces, Vol. I, North-Holland, Amsterdam, 2001, 233-269.

[6] A. P. Calderón, Ergodic theory and translation-invariant operators, Proc. Nat. Acad. Sci. U.S.A. 59 (1968), 349-353.

[7] W. Chojnacki, Group representations of bounded cosine functions, J. Reine Angew. Math. 478 (1996), 61-84.

[8] —, On group decompositions of bounded cosine sequences, Studia Math. 181 (2007), 61-85.

[9] I. Cioranescu and V. Keyantuo, On operator cosine functions in UMD spaces, Semigroup Forum 63 (2001), 429-440.

[10] R. R. Coifman and G. Weiss, Transference Methods in Analysis, CBMS Reg. Conf. Ser. Math. 31, Amer. Math. Soc., Providence, RI, 1976.

[11] R. E. Edwards and G. I. Gaudry, Littewood-Paley and Multiplier Theory, Springer, Berlin, 1977.

[12] H. O. Fattorini, Some remarks on second-order abstract Cauchy problems, Funkcial. Ekvac. 24 (1981), 331-344.

[13] - The Cauchy Problem, Encyclopedia Math. Appl. 18, Addison-Wesley, Reading, MA, 1983.

[14] —, Second Order Linear Differential Equations in Banach Spaces, North-Holland, Amsterdam, 1985.

[15] M. Haase, The group reduction for bounded cosine functions on UMD spaces, Math. Z. 262 (2009), 281-299.

[16] J. Kisyński, On operator-valued solutions of d'Alembert's functional equation I, Colloq. Math. 23 (1971), 107-114.

[17] - On cosine operator functions and one-parameter groups of operators, Studia Math. 44 (1972), 93-105.

[18] —, On operator-valued solutions of d'Alembert's functional equation II, ibid. 42 (1972), 43-66.

[19] S. G. Kreĭn, Linear Differential Equations in Banach Space, Nauka, Moscow, 1967 (in Russian); English transl.: Transl. Math. Monogr. 29, Amer. Math. Soc., Providence, RI, 1971. 
[20] S. B. Stechkin, On bilinear forms, Dokl. Akad. Nauk SSSR (N.S.) 71 (1950), 237-240 (in Russian).

[21] S. Tymowski, On the analogue of the formula $\cos t=\frac{1}{2} e^{i t}+\frac{1}{2} e^{-i t}$ for operator cosine functions, Comment. Math. Prace Mat. 23 (1983), 173-182.

Wojciech Chojnacki

School of Computer Science

The University of Adelaide

Adelaide, SA 5005, Australia

E-mail: wojciech.chojnacki@adelaide.edu.au

and

Wydział Matematyczno-Przyrodniczy

Szkoła Nauk Ścisłych

Uniwersytet Kardynała Stefana Wyszyńskiego

Dewajtis 5

01-815 Warszawa, Poland 